\title{
Intervenção de musicoterapia nos sinais vitais de crianças com transtorno do
}

\section{espectro autista: Estudo piloto}

\author{
Music therapy intervention in the vital signs of children with autistic spectrum disorder: Pilot study \\ Intervención de musicoterapia en los signos vitales de niños con trastorno del espectro autista:
}

Estudio piloto

Recebido: 13/03/2021 | Revisado: 20/03/2021 | Aceito: 20/03/2021 | Publicado: 28/03/2021

Juliana Macêdo Magalhães

ORCID: http://orcid.org/0000-0001-9547-9752 Centro Universitário UNINOVAFAPI, Brasil E-mail: juliana.magalhaes@uninovafapi.edu.br

Pedro Venicius de Sousa Batista

ORCID: http://orcid.org/0000-0002-9441-0996 Centro Universitário UNINOVAFAPI, Brasil E-mail: pedroveni@outlook.com

Emilia Angela Lo Schiavo Arisawa

ORCID: http://orcid.org/0000-0003-3526-3890 Universidade do Vale do Paraíba, Brasil E-mail: mirela@univap.br

\begin{abstract}
Resumo
Objetivo: Avaliar os sinais vitais de crianças com transtorno do espectro autista no contexto de intervenção de musicoterapia. Métodos: Estudo piloto experimental de intervenção de musicoterapia, desenvolvido no nordeste do Brasil. Sete crianças com transtorno do espectro autista foram avaliadas, distribuída em três para intervenção de musicoterapia e quatro sem intervenção. Os dados foram analisados utilizando testes não paramétricos. Resultados: As crianças apresentavam média de idade de oito anos, com maioria do sexo masculino. Descritivamente houve redução nos sinais vitais de $3,7 \%$ na saturação de $\mathrm{O}_{2}$, diminuição de $3,3 \mathrm{mmHg}$ na pressão arterial sistólica e aumento de 5,0 bpm na frequência cardíaca no grupo que passou por intervenção de musicoterapia. $\mathrm{O}$ grupo sem intervenção apresentou aumento médio de $0,5 \%$ na saturação de $\mathrm{O}_{2}$ e de $2,5 \mathrm{mmHg}$ na pressão arterial diastólica. Conclusão: Conclui-se que os efeitos da musicoterapia nos sinais vitais foram indicativos de respostas satisfatórias para as crianças com autismo.
\end{abstract}

Palavras-chave: Transtorno do espectro autista; Musicoterapia; Sinais vitais.

\begin{abstract}
Objective: To evaluate the vital signs of children with autism spectrum disorder in the context of music therapy intervention. Methods: Experimental pilot study of music therapy intervention, developed in northeastern Brazil. Seven children with autism spectrum disorder were evaluated, distributed in three for music therapy intervention and four without intervention. The data were analyzed using non-parametric tests. Results: The children had an average age of eight years, with a majority of males. Descriptively, there was a 3.7\% reduction in vital signs in $\mathrm{O} 2$ saturation, a decrease of $3.3 \mathrm{mmHg}$ in systolic blood pressure and an increase of $5.0 \mathrm{bpm}$ in heart rate in the group that underwent music therapy intervention. The group without intervention showed an average increase of $0.5 \%$ in $\mathrm{O} 2$ saturation and $2.5 \mathrm{mmHg}$ in diastolic blood pressure. Conclusion: It is conclude effects of music therapy on vital signs were indicative of satisfactory responses for children with autism.
\end{abstract}

Keywords: Autistic spectrum disorder; Music therapy; Vital signs.

\section{Resumen}

Objetivo: Evaluar los signos vitales de niños con trastorno del espectro autista en el contexto de la intervención de musicoterapia. Métodos: Estudio piloto experimental de intervención de musicoterapia, desarrollado en el noreste de Brasil. Se evaluaron siete niños con trastorno del espectro autista, distribuidos en tres para la intervención de musicoterapia y cuatro sin intervención. Los datos se analizaron mediante pruebas no paramétricas. Resultados: Los niños tenían una edad promedio de ocho años, con una mayoría de varones. Descriptivamente, hubo una reducción del $3.7 \%$ en los signos vitales en la saturación de $\mathrm{O} 2$, una disminución de $3.3 \mathrm{mmHg}$ en la presión arterial sistólica y un aumento de $5.0 \mathrm{lpm}$ en la frecuencia cardíaca en el grupo que se sometió a la intervención de musicoterapia. El grupo sin intervención mostró un aumento promedio de $0.5 \%$ en la saturación de $\mathrm{O} 2$ y $2.5 \mathrm{mmHg}$ en la presión arterial 
diastólica. Conclusión: Se concluye los efectos de la musicoterapia sobre los signos vitales indicaron respuestas satisfactorias para los niños con autismo.

Palabras clave: Trastorno del espectro autista; Terapia musical; Signos vitales.

\section{Introdução}

A música produz diferentes estímulos psíquicos no indivíduo, atuando no seu estado mental e emocional. Intervenções musicais podem ter efeito positivo e promover mudanças comportamentais mensuráveis em crianças com autismo, constituindo um ponto forte para o auxílio terapêutico potencial desses indivíduos (Sharda et al., 2018). No entanto, a evidência da música como terapia ainda é limitada e não há comprovação que a musicoterapia poderá relaxar a criança com Transtorno do Espectro Autista (TEA) a ponto de refletir alterações nos sinais vitais, o que torna importante a sua investigação.

As abordagens terapêuticas têm objetivado o desenvolvimento de pessoas com limitações de caráter de interação social, desempenho cognitivo e comportamental relacionado. Nesse sentido, notabiliza-se a relevância de fatores associados as tais abordagens terapêuticas em crianças com TEA. Dentre as abordagens utilizadas destaca-se a musicoterapia para promoção da saúde do cliente por meio de experiências musicais (Mossler et al., 2019).

De forma geral, experiências musicais estão presentes no convívio entre pais, crianças e seus pares desde a primeira infância, de forma espontânea. O canto e as brincadeiras musicais atraem a atenção das crianças, sensibilizando-as por meio dos sons e potencializando seus níveis de concentração. Destaca-se ainda que, além dos benefícios sociais da música para crianças e famílias com desenvolvimento típico, há interesse em aproveitar a música para fins terapêuticos em crianças com TEA (Lense \& Camarata, 2020).

Nesse contexto, a musicoterapia surge como uma abordagem significativa no tratamento do TEA, que promove experiências baseadas nas expressões e interesses do cliente, alcançando necessidades físicas, mentais, sociais, emocionais e cognitivas. Outrossim, a musicoterapia é considerada uma intervenção precoce promissora para crianças pequenas com TEA e suas famílias, estabelecendo uma relação de comunicação, expressão e mobilização que favorece o efeito terapêutico (Mossler et al., 2019).

A musicoterapia está fundamentada no uso da música ou de elementos musicais por um profissional capacitado, com o intuito de promover e colaborar com a saúde do indivíduo. Estudos empíricos e pesquisas clínicas afirmam que os indivíduos com TEA podem se beneficiar com a musicoterapia, no que diz respeito à interação social, linguagem, comunicação e comportamento. Nesse tocante, percebe-se que crianças autistas são atraídas pela música e respondem melhor à música do que às palavras (Sharda et al., 2018; Raberon et al., 2020; Sharda et al., 2019).

Assim, a intervenção de musicoterapia compreende uma importante abordagem popular para crianças com TEA, cujas evidências científicas encontram-se em constante avanço, existindo, porém, limitações metodológicas que reduzem o potencial de generalização dos resultados obtidos e que podem predizer seu real efeito nas habilidades sociais. Por isso, ressalta-se a importância de estudar mecanismos de mudança que as experiências musicais podem proporcionar para o desenvolvimento de crianças com TEA (Schmid et al., 2020), com destaque para a relação com a variação de sinais vitais durante a abordagem terapêutica.

Dessa forma, é essencial a promoção de maior engajamento e produção de estudos voltados para esse tema, devido à necessidade da identificação dos mecanismos e alterações fisiológicas que ocorrem a partir da musicoterapia. Assim, este estudo teve como objetivo avaliar os sinais vitais de crianças com TEA no contexto de intervenção de musicoterapia. 


\section{Metodologia}

Estudo piloto experimental de intervenção de musicoterapia, desenvolvido em uma Associação de Amigos dos Autistas (AMA) localizada no Nordeste do Brasil, no período de fevereiro a março de 2020. A população do estudo compreendeu 30 crianças com diagnóstico de TEA atendidas nessa associação.

Para composição da amostra, foram critérios de inclusão: possuir idade de um a nove anos e apresentar escore 30 a 36,5 pontos da escala de Childhood Autism Rating Scale (CARS), que consiste nos níveis leve e moderado. Foram critérios de exclusão: apresentar disritmia, estar em uso de medicamentos que afetam os vasos sanguíneos, anti-inflamatórios não esteroides ou corticosteroides, apresentar processos infecciosos, doenças respiratórias e do aparelho auditivo.

Assim, a partir dos critérios de inclusão a randomização das crianças foram alocadas em dois grupos por meio do programa Random Allocation Software estando pré-determinado que os três primeiros nomes fictícios integraram ao grupo de intervenção de musicoterapia e os demais fizeram parte do grupo sem intervenção.

Foi utilizado um formulário de coleta, contendo dados sociodemográficos (idade, sexo, raça, escolaridade, renda familiar, familiares na residência, local de residência, uso de medicação, tempo na associação e atividades que desenvolviam) e sinais vitais (antes e depois, conforme intervalo determinado para a intervenção). Para triagem, foi verificado o diagnóstico de TEA e a pontuação da escala CARS, por meio dos seguintes critérios: relações pessoais, imitação, resposta emocional, uso corporal, uso de objetos, resposta a mudanças, resposta visual, resposta auditiva, resposta e uso do paladar, olfato e tato, medo ou nervosismo, comunicação verbal, comunicação não verbal, nível de atividade, nível e consistência da resposta intelectual e impressões gerais). O contato com as crianças e seus pais foi realizado na própria instituição, com agendamento prévio das datas e horários do atendimento, conforme disponibilidade dos participantes e de seus responsáveis.

Após obtenção do consentimento, a coleta de informações foi realizada em sala reservada da instituição. A intervenção do estudo foi a musicoterapia de abordagem improvisacional, que consiste em fazer musical livre a partir da voz, movimentos ou instrumentos musicais (Crawford et al., 2017), aplicada na forma de livre improvisação, onde o indivíduo escolhe as estruturas rítmicas, melodias ou harmonia de forma que considerar melhor (Bieleninik et al., 2017). O estudo foi desenvolvido conforme protocolo de atendimento de musicoterapia improvisacional músico-centrada para crianças com autismo (Gattino, 2015).

Desta forma, na primeira etapa, ocorreu o contato, ambientação e exploração, assim o musicoterapeuta cantou uma canção de "oi" e convidou a criança para entrar na sala, tocar os instrumentos (violão, teclado, flauta doce soprano e variados instrumentos de percussão de pequeno porte). Em seguida, a criança foi estimulada a explorar o espaço e os instrumentos, o musicoterapeuta aguardou a iniciativa sonora da criança, e interveio musicalmente, convidando o paciente a se engajar na atividade musical.

Na segunda etapa, o musicoterapeuta sustentou a expressão musical da criança e conduziu as improvisações, para que juntos mantivessem a comunicação musical. Na terceira etapa, o musicoterapeuta propôs frases musicais elaboradas, consolidação e ampliação dos temas clínicos. Após esse momento, consistiu na canção do "tchau" e na guarda dos instrumentos. A intervenção de musicoterapia compreendeu oito sessões, realizadas duas vezes por semana, e cada sessão teve duração média de 50 minutos. A coleta dos dados de caracterização e triagem foi realizada com os pais/responsáveis e a mensuração dos parâmetros vitais foi operacionalizada com esfigmomanômetro aneroide e oxímetro portátil At101c KIDS Bioland.

Os dados foram processados no software IBM SPSS, versão 26.0. Foram calculadas estatísticas descritivas (média e desvio padrão, mediana e intervalo interquartil, para as variáveis quantitativas; e frequências, para as qualitativas). Foram calculadas as diferenças entre as medidas de saturação de $\mathrm{O}_{2}$, frequência cardíaca e pressão arterial sistólica e diastólica (diferença $=$ depois - antes). Na análise inferencial, foram utilizados testes não paramétricos: teste de Mann-Whitney para 
comparação de características e das diferenças entre as distribuições dos parâmetros fisiológicos dos grupos (musicoterapia vs. sem intervenção musical), teste de Wilcoxon para comparação dos parâmetros fisiológicos antes e depois de cada grupo, e teste Likelihood Ratio (amostra < 25 participantes) para as associações. Foram apresentados os rankings dos grupos, conforme as diferenças entre as medidas de saturação de $\mathrm{O}_{2}$, frequência cardíaca e pressão arterial sistólica e diastólica. Todas as análises foram realizadas com nível de significância de $5 \%$.

$\mathrm{O}$ estudo atendeu às normatizações nacionais e internacionais para pesquisas com seres humanos. Foi aprovado por Comitê de Ética em Pesquisa, sob parecer n. ${ }^{\circ} 3.604 .288$ e CAAE: 20662719.9.0000.5210. Foram obtidos os consentimentos para participação na pesquisa por meio da assinatura do termo de consentimento livre e esclarecido (responsáveis) e termo de assentimento livre e esclarecido (participantes).

\section{Resultados}

A amostra, em sua totalidade, apresentava diagnóstico de autismo infantil (CID 10 - F84.0), de grau leve a moderado 7 (100,0\%). Apenas uma criança era diagnosticada também com Distúrbio desafiador e de oposição (CID 10 - F91.3). As características das crianças que compuseram a amostra estão apresentadas na (Tabela 1).

Tabela 1. Caracterização das crianças com transtorno do espectro autista (n=7). Teresina, PI, Brasil, 2020.

\begin{tabular}{|c|c|c|c|c|}
\hline Característica & Musicoterapia $(n=3)$ & Sem intervenção $(n=4)$ & Total $(n=7)$ & $p$ \\
\hline Idade $(\mathrm{M} \pm \mathrm{DP})$ & $7,9( \pm 2,3)$ & $7,5( \pm 1,9)$ & $8,0( \pm 2,0)$ & $0,400^{u}$ \\
\hline Sexo & & & & $0,166^{q}$ \\
\hline Masculino & $2(66,7 \%)$ & $4(100,0 \%)$ & $6(85,7 \%)$ & \\
\hline Feminino & $1(33,3 \%)$ & - & $1(14,3 \%)$ & \\
\hline Cor/raça & & & & $0,658^{q}$ \\
\hline Parda & $2(66,7 \%)$ & $2(50,0 \%)$ & $4(57,1 \%)$ & \\
\hline Branca & $1(33,3 \%)$ & $2(50,0 \%)$ & $3(42,9 \%)$ & \\
\hline Escola & & & & $0,265^{q}$ \\
\hline Sim & $3(100 \%)$ & $3(75,0 \%)$ & $6(85,7 \%)$ & \\
\hline Não & - & $1(25,0 \%)$ & $1(14,3 \%)$ & \\
\hline Renda familiar mensal & & & & $0,102^{q}$ \\
\hline 1 salário mínimo & $1(33,3 \%)$ & $4(100,0 \%)$ & $5(71,4 \%)$ & \\
\hline 1-2 salários mínimos & $1(33,3 \%)$ & - & $1(14,3 \%)$ & \\
\hline Superior a 3 salários mínimos & $1(33,3 \%)$ & - & $1(14,3 \%)$ & \\
\hline Familiares na residência & & & & $0,148^{q}$ \\
\hline Mãe e Pai & - & $2(50,0 \%)$ & $2(28,6 \%)$ & \\
\hline Mãe, pai e irmão(s) & $1(33,3 \%)$ & $1(25,0 \%)$ & $2(28,6 \%)$ & \\
\hline Mãe, irmão e avós & - & $1(25,0 \%)$ & $1(14,3 \%)$ & \\
\hline Mãe, avós e tias & $1(33,3 \%)$ & - & $1(14,3 \%)$ & \\
\hline Avó & $1(33,3 \%)$ & - & $1(14,3 \%)$ & \\
\hline Local de residência & & & & $0,166^{q}$ \\
\hline Teresina & $2(66,7 \%)$ & $4(100,0 \%)$ & $6(85,7 \%)$ & \\
\hline Timon & $1(33,3 \%)$ & - & $1(14,3 \%)$ & \\
\hline Tempo na AMA & & & & $0,396^{q}$ \\
\hline 1 mês & - & $1(25,0 \%)$ & $1(14,3 \%)$ & \\
\hline 2 anos & $2(66,7 \%)$ & $1(25,0 \%)$ & $3(42,9 \%)$ & \\
\hline 5 anos & $1(33,3 \%)$ & $1(25,0 \%)$ & $2(28,6 \%)$ & \\
\hline$>5$ anos & - & $1(25,0 \%)$ & $1(14,3 \%)$ & \\
\hline Faz usode medicação & & & & $0,092^{q}$ \\
\hline $\operatorname{Sim}$ & - & $2(50,0 \%)$ & $2(28,6 \%)$ & \\
\hline Não & $3(100,0 \%)$ & $2(50,0 \%)$ & $5(71,4 \%)$ & \\
\hline
\end{tabular}

Legenda: M \pm DP: média \pm desvio padrão; $p$ : significância do teste; $u$ : teste de Mann-Whitney; $q$ : teste Likelihood Ratio; AMA: Associação de Amigos dos Autistas. Fonte: Autores (2020). 
As crianças com TEA que compuseram a amostra deste estudo apresentaram média ( \pm desvio padrão) de idade de 8,0 $( \pm 2,0)$ anos, sem diferença significativa entre os grupos $(\mathrm{p}=0,400)$. De modo geral, predominaram crianças do sexo masculino $6(85,7 \%)$, pardas $4(57,1 \%)$, que frequentavam a escola $6(85,7 \%)$, cursando os níveis fundamental $5(83,3 \%)$ e infantil 1 (16,7\%), cujas famílias informaram possuir renda de um salário mínimo 5 (71,4\%), moravam com os pais 4 (57,1\%), residentes em área urbana da capital $6(85,7 \%)$, sendo acompanhados na AMA há, pelo menos, dois anos 6 (85,7\%). A maioria não utilizava medicações $5(71,4 \%)$ (tabela 1$)$. A (Figura 1) apresenta as atividades regularmente realizadas pelas crianças na AMA.

Figura 1. Atividades desenvolvidas pelas crianças com transtorno do espectro autista (n=7). Teresina, PI, Brasil, 2020.

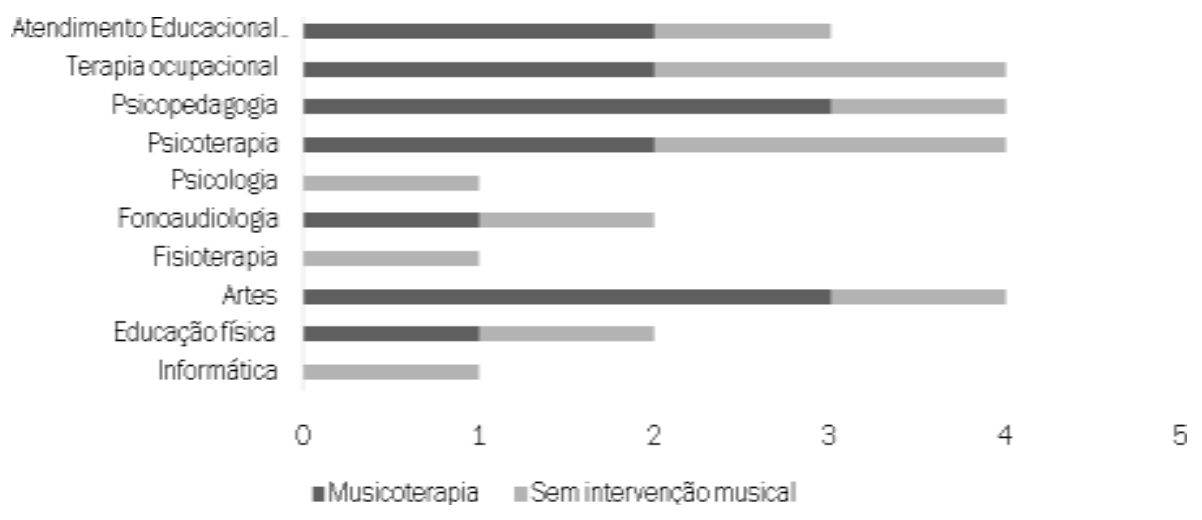

Fonte: Autores (2020).

As atividades mais predominantes foram terapia ocupacional $4(57,1 \%)$, psicopedagogia $4(57,1 \%)$, psicoterapia 4 $(57,1 \%)$ e artes $4(57,1 \%)$, seguido de atendimento educacional especializado $3(42,9 \%)$, com menor frequência de crianças que passaram pela intervenção de musicoterapia. Psicologia 1 (14,3\%), fisioterapia 1 (14,3\%) e informática 1 (14,3\%) foram atividades terapêuticas desenvolvidas somente por crianças do grupo que não participou de intervenção musical (figura 1). Apenas $3(42,9 \%)$ utilizavam medicação de uso contínuo, porém, todas as crianças não usaram recentemente algum medicamento em relação ao momento de aferição dos sinais vitais 7 (100,0\%) ou não ingeriram cafeína na última hora 7 $(100,0 \%)$. Somente $2(28,6 \%)$ haviam realizado exercícios, porém, com intervalo superior a 60 minutos. A (Tabela 2) descreve as medidas de saturação de $\mathrm{O}_{2}$, frequência cardíaca e pressão arterial das crianças nas etapas do estudo. 
Tabela 2. Sinais vitais antes e depois das crianças com transtorno do espectro autista (n=7). Teresina, PI, Brasil, 2020.

\begin{tabular}{|c|c|c|c|c|c|c|c|}
\hline \multirow[b]{2}{*}{ Parâmetro } & \multicolumn{3}{|c|}{ Musicoterapia } & \multicolumn{3}{|c|}{ Sem intervenção } & \multirow[b]{2}{*}{$\boldsymbol{P}$} \\
\hline & Antes & Depois & $\begin{array}{c}\text { Diferença } \\
\text { média }\end{array}$ & Antes & Depois & $\begin{array}{c}\text { Diferença } \\
\text { média }\end{array}$ & \\
\hline Saturação de $\mathrm{O}_{2}$ & & & $-3,7$ & & & 0,5 & 0,705 \\
\hline $\mathrm{M} \pm \mathrm{DP}$ & $98,3 \pm 1,2$ & $94,7 \pm 4,9$ & & $97,5 \pm 0,6$ & $98,0 \pm 0,8$ & & \\
\hline $\mathrm{Md} \pm \mathrm{IIQ}$ & $99,0 \pm 2,0$ & $97,0 \pm 8,0$ & & $97,5 \pm 1,0$ & $98,0 \pm 1,5$ & & \\
\hline FC & & & 5,0 & & & 0,0 & 0,416 \\
\hline $\mathrm{M} \pm \mathrm{DP}$ & $88,7 \pm 13,8$ & $93,7 \pm 13,5$ & & $101,8 \pm 9,4$ & $101,8 \pm 8,0$ & & \\
\hline $\mathrm{Md} \pm \mathrm{IIQ}$ & $94,0 \pm 26,0$ & $94,0 \pm 13,0$ & & $102,0 \pm 17,3$ & $104,5 \pm 13,8$ & & \\
\hline PAS & & & $-3,3$ & & & 0,0 & 0,564 \\
\hline $\mathrm{M} \pm \mathrm{DP}$ & $93,3 \pm 5,8$ & $90,0 \pm 10,0$ & & $100,0 \pm 14,1$ & $100,0 \pm 18,3$ & & \\
\hline $\mathrm{Md} \pm \mathrm{IIQ}$ & $90,0 \pm 25,0$ & $90,0 \pm 35,0$ & & $105,0 \pm 25,0$ & $100,0 \pm 35,0$ & & \\
\hline PAD & & & 0,0 & & & 2,5 & 0,655 \\
\hline $\mathrm{M} \pm \mathrm{DP}$ & $63,3 \pm 8,8$ & $63,3 \pm 5,8$ & & $62,5 \pm 5,0$ & $65,0 \pm 17,3$ & & \\
\hline $\mathrm{Md} \pm \mathrm{IIQ}$ & $60,0 \pm 10,0$ & $60,0 \pm 10,0$ & & $60,0 \pm 7,5$ & $60,0 \pm 30,0$ & & \\
\hline
\end{tabular}

Legenda: $\mathrm{M} \pm$ DP: média \pm desvio padrão; $\mathrm{Md} \pm$ IIQ: mediana \pm intervalo interquartil; FC: frequência cardíaca; PAS: pressão arterial sistólica; PAD: pressão arterial diastólica; $p$ : significância do teste de Mann-Whitney. Fonte: Autores (2020).

Não foram verificadas diferenças estatisticamente significativas entre as distribuições de dados de saturação de $\mathrm{O}_{2}$ $(\mathrm{p}=0,705)$, frequência cardíaca $(\mathrm{p}=0,416)$, pressão arterial sistólica $(\mathrm{p}=0,564)$ e diastólica $(\mathrm{p}=0,655)$ em ambos os grupos. Entretanto, as médias das diferenças entre as medidas pós e pré, descritivamente, indicaram a redução de $3,7 \%$ na saturação de $\mathrm{O}_{2}$, diminuição de $3,3 \mathrm{mmHg}$ na pressão arterial sistólica e aumento de 5,0 bpm na frequência cardíaca no grupo que passou por intervenção de musicoterapia. O grupo sem intervenção apresentou, também descritivamente, aumento médio de $0,5 \%$ na saturação de $\mathrm{O}_{2}$ e de $2,5 \mathrm{mmHg}$ na pressão arterial diastólica, conforme apresentado na (Tabela 2). Os rankings dos participantes para os sinais vitais avaliados estão demonstrados na (Figura 2). 
Figura 2. Ranking da comparação antes e depois das crianças com transtorno do espectro autista (n=7). Teresina, PI, Brasil, 2020

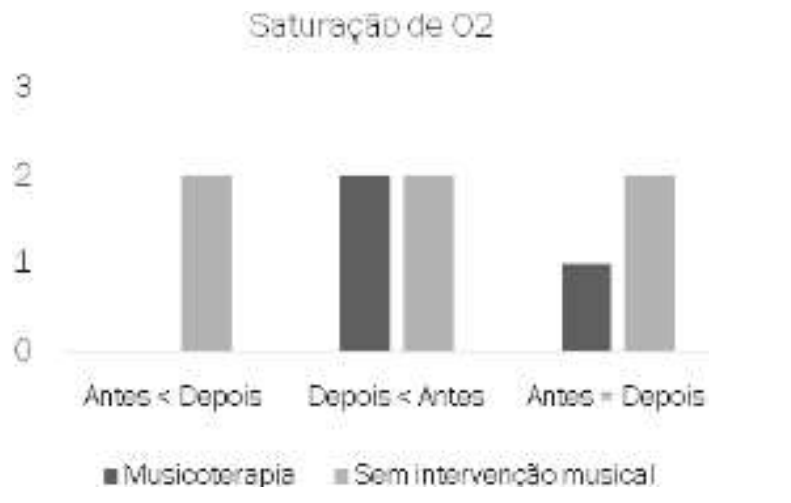

Pressāo arterial sistốlica

3

2

1 0

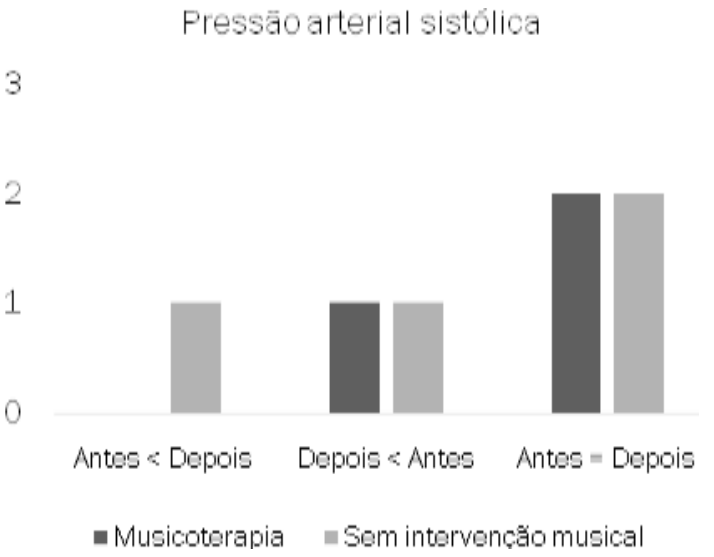

Frequencia cardiaca

3

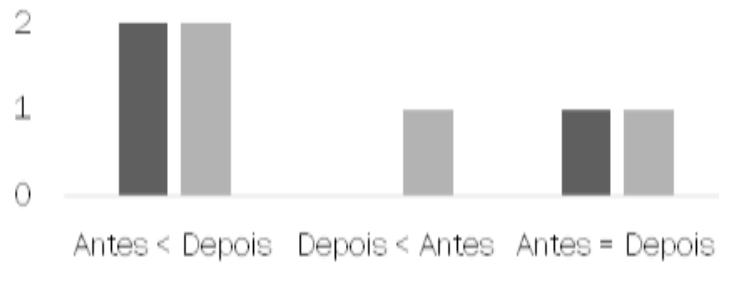

- Musicoterapia = Sem intervençăo musical

Pressāo arterial diastólica

3

2

1

0

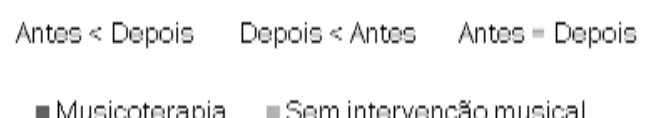

Fonte: Autores (2020).

A saturação de $\mathrm{O} 2$ apresentou aumento apenas no grupo sem intervenção. Aumento na frequência cardíaca (antes < depois) foi verificado em ambos os grupos. Verificou-se aumento da pressão arterial sistólica apenas no grupo que não recebeu intervenção, enquanto a pressão diastólica permaneceu em equilíbrio somente no grupo da musicoterapia, conforme a (Figura 2).

\section{Discussão}

A predominância de crianças do sexo masculino na amostra corrobora com a literatura geral sobre TEA. O autismo é considerado quatro vezes mais comum no sexo masculino, dado reforçado por um estudo do Centers for Disease Control and Prevention, que caracteriza sua prevalência na razão de 4:1, comparando-se o sexo masculino e o feminino (Rocha, Souza, Costa, \& Portes, 2019). Essa diferença é comumente explicada pela influência genética, em que a testosterona circulante se liga a receptores cerebrais, aumentando a excitação cerebral e tornando o sexo masculino mais vulnerável ao TEA. Outra explicação envolve regiões do cromossomo Y, presente em indivíduos do sexo masculino, que possui alguns genes específicos que podem favorecer o desenvolvimento de TEA (Reis, Neder, Moraes, \& Oliveira, 2019).

A idade média das crianças abordadas retoma a forte apresentação de traços característicos do transtorno. Além disso, quase a totalidade frequentava a escola, cujo ambiente pode se apresentar como importante elemento para integração social dessas crianças. No entanto, percebe-se que as crianças do estudo estão com atraso no desenvolvimento escolar, considerandose que a maioria ainda se encontrava no ensino infantil ou cursando as primeiras séries do fundamental. 
A Educação Infantil apresenta-se como um dos pilares iniciais de socialização e aprendizagem além do contexto familiar, o que reforça a relevância da inclusão escolar de crianças com autismo, uma vez que essa integração proporciona experiências, favorece o desenvolvimento e a interação social nessa fase da vida. Ademais, interações sociais entre as crianças valorizam a multiplicidade, de forma a transpor as barreiras atitudinais e comunicacionais (Agripino Ramos, Lemos, \& Salomao, 2019).

Todavia, o sistema educacional ainda não oferta o serviço adequado para crianças com TEA. Estudos apontam o despreparo dos profissionais para ensinar e educar esses indivíduos tem influência de uma formação insuficiente, por falta de aprofundamento sobre o quadro de crianças com TEA, o que pode estar relacionado ao fato de crianças com autismo permanecerem por mais tempo na escola, representando atraso no desenvolvimento escolar. Além disso, alguns pais de crianças com TEA acreditam que o tempo em ambiente escolar não é suficiente e proveitoso para o desenvolvimento de habilidades, como estimulação cognitiva, de linguagem, e de aprendizado pedagógico (Campos \& Fernandes, 2016). Além disso, fatores como dificuldade na socialização, organização, distração e dificuldade em sequenciar são problemas encontrados por crianças com TEA em ambientes escolares, dificultando ainda mais o seu desenvolvimento (Ferreira \& França, 2017)

No presente estudo, a maioria das crianças com TEA residia com os pais, sendo que o principal cuidador era a mãe, que foi responsável pelo compartilhamento de informações sobre as crianças ao longo do desenvolvimento do estudo. Apenas um caso foi abordado com a avó. Esse dado é similar à literatura, que aponta que o papel de cuidar dos filhos, principalmente portadores de necessidades especiais, é destinado às mães ou, de maneira geral, às mulheres. Dificuldades apresentadas por portadores de TEA, devido à sua cronicidade, condições físicas e mentais, resultam em uma maior dependência em relação às suas mães. Ademais, as mães têm mais provimento em cuidar dos filhos, enquanto os pais se mantêm na retaguarda e ficam encarregado pelo sustento financeiro de sua família (Mapelli et al., 2018).

De modo geral, as crianças com TEA que compuseram a amostra deste estudo apresentaram uma boa frequência de participação, estando envolvidas em duas ou mais atividades terapêuticas. Apenas duas crianças utilizavam medicação, mas que não interferiam nos sinais vitais. É importante destacar que, na associação onde o estudo foi desenvolvido, as atividades envolvendo música tratavam apenas de musicalidade sem o recurso terapêutico e, no momento da coleta, encontrava-se inativa há algum tempo por ausência de profissional para conduzi-la e talvez, por isso, destacaram-se a terapia ocupacional, psicopedagogia, psicoterapia e artes. O fato de a maioria receber acompanhamento pela AMA por um período prolongado reforça a presença de respostas positivas a partir das intervenções em geral na vivência e na qualidade de vida das crianças com TEA.

Nesse contexto, constata-se que algumas práticas pedagógicas e atividades terapêuticas não verbais vêm ganhando espaço e estabelecendo importante papel na qualidade de vida das crianças com TEA, sendo consideradas como parte do tratamento para esses indivíduos. Dentre os benefícios dessas práticas, há o desenvolvimento na comunicação verbal, alfabetização, integração social e outras habilidades de acordo com o grau de comprometimento, intensidade e adequação desses métodos. Essas práticas não verbais são realizadas por uma equipe multidisciplinar, que busca desenvolver formas de comunicação e expressão dos indivíduos com TEA (Lima, Gehres, Lorenzini, \& Brasileiro, 2017).

As crianças que participaram da intervenção de musicoterapia apresentaram redução de $3,7 \%$ na saturação de $\mathrm{O}_{2}$, no presente estudo. No TEA, é comum a dificuldade de respirar profundamente e estudos destacaram algumas manifestações, como hipoperfusão cerebral, estresse oxidativo e disfunção mitocondrial, que favorecem uma menor saturação, em geral, de oxigênio (Rossignol \& Frye, 2012; Sakulchit, Ladish, \& Goldman, 2017). Diante disso, por exemplo, algumas pesquisas têm sido desenvolvidas para verificar a eficácia da oxigenoterapia hiperbárica, técnica que fornece uma maior concentração de oxigênio por meio de uma câmara ou tubo contendo pressão atmosférica superior ao nível do mar. Em uma amostra de seis crianças autistas ( 2 a 7 anos de idade), foi reportada melhora estatisticamente significativa de sintomas clínicos após 40 sessões 
com duração de um hora de oxigenoterapia (Rossignol \& Rossignol, 2006). Na Tailândia, em estudo realizado com sete crianças com TEA (5 a 9 anos de idade), cinco apresentaram melhorias em domínios físicos e sociais após 10 sessões (Chungpaibulpatana et al., 2008). Em amostras maiores, de crianças acima de cinco anos e com gravidade inicial de autismo mais baixa, a oxigenoterapia foi confirmada como um tratamento seguro e bem tolerado (Rossignol et al., 2009), o que reforça a importância da avaliação da saturação de $\mathrm{O}_{2}$ nesse público-alvo.

Embora a redução da saturação de $\mathrm{O}_{2}$ neste estudo pareça um fator negativo da intervenção de musicoterapia realizada, por outro lado, é preciso destacar que crianças com TEA possuem um padrão de comportamento mais sensível a elementos estressores do ambiente, que podem levar ao aumento da saturação de $\mathrm{O}_{2}$, em decorrência da hiperventilação proveniente de agitação psicomotora e aumento dos níveis de adrenalina (Panerai et al., 2020; Le Menn-Tripi et al., 2019). Esses fatores podem ser ainda mais relevantes, considerando-se que a amostra do estudo é proveniente de um país em desenvolvimento, que possui menor renda e possivelmente está exposta a mais elementos dificultadores. Assim, os resultados obtidos nesta pesquisa demonstram que a musicoterapia relaxou as crianças, enquanto o grupo que não teve intervenção apresentou aumento médio de $0,5 \%$ na saturação de $\mathrm{O}_{2}$, o que pode indicar maior suscetibilidade aos estressores do ambiente, sem a musicoterapia.

Além disso, crianças que realizaram musicoterapia tiveram um aumento médio de 5,0 bpm na frequência cardíaca comparada a nenhuma variação nas crianças sem intervenção. Em geral, indivíduos com TEA possuem frequência cardíaca mais baixa do que o esperado, possivelmente devido a um déficit no sistema límbico (Tyler, MacDonald, \& Menear, 2014). Logo, o achado neste estudo foi interessante, tendo em vista que esse sinal vital facilmente sofre variação ao longo do dia e a musicoterapia parece ter proporcionado envolvimento emocional. Isso permitiu que as crianças expressassem também suas emoções, quando colocadas suas músicas de preferência, de modo a refletir positivamente na variação da frequência cardíaca.

Em relação à pressão arterial, o grupo que realizou musicoterapia teve redução da sistólica e manutenção da diastólica, enquanto as crianças sem intervenção mantiveram a sistólica e aumentaram à diastólica. Denota-se, aqui, o potencial que a música apresenta em influenciar e produzir alterações no corpo humano, gerando diversas emoções, capazes de provocar estado de ansiedade ou, ao contrário, relaxamento e libertação. Nesse processo, o sistema cardiovascular apresenta-se como indicador do estado funcional do cérebro humano e as alterações rítmicas cardíacas resultam do desenvolvimento do processo nervoso do indivíduo (Volokitina \& Ivanitskaya, 2005).

Dessa forma, percebe-se que o sistema cardiovascular é sensível a uma variedade de estados psicológicos e comportamentais, e os sinais vitais relacionados podem ser importantes variáveis dependentes na avaliação de intervenções, como a musicoterapia. No tocante à musicoterapia, as experiências proporcionaram alterações em parâmetros fisiológicos das crianças e parece existir uma influência importante na redução e controle da pressão arterial, que promove o relaxamento corporal, contribuindo para a diminuição de reações a elementos estressores, com consequente melhoria da qualidade de vida das crianças com TEA.

\section{Conclusão}

Conclui-se que o estudo permitiu identificar, descritivamente, efeitos da musicoterapia nos sinais vitais, indicativos de respostas satisfatórias para as crianças com TEA, comparativamente às que não tiveram intervenção. Constatou-se que, para o grupo de intervenção, houve redução nos níveis de saturação de $\mathrm{O}_{2}$ e pressão arterial sistólica, além de aumento da frequência cardíaca, que foram benéficos para as crianças autistas na medida em que apresentaram distanciamento de estressores ambientais e aparente resposta emocional conforme o direcionamento da musicoterapia.

Uma limitação do estudo foi a pequena amostra, que dificultou evidenciar inferencialmente as diferenças encontradas, além de reduzir o potencial de generalização dos resultados. Além disso, a amostra foi limitada diante do pequeno número de 
crianças assistidas na associação foco do estudo, somado à dificuldade de acesso devido ao início do alerta quanto à pandemia de coronavírus 2019 (COVID-19) no Brasil.

Embora a musicoterapia contribua para o desenvolvimento social e desempenho cognitivo de crianças com TEA, a evidência dessa abordagem terapêutica ainda é limitada e são escassas comprovações quanto à eficácia no relaxamento a ponto de refletir nos sinais vitais e atingir seus objetivos. Nesse sentido, os dados deste estudo sugeriram a existência de uma relação com a variação de sinais vitais a partir da abordagem terapêutica por meio da musicoterapia, o que abre pressuposto para realização de avaliações com amostras maiores para somar esforços na promoção da saúde de crianças com transtorno do espectro autista por meio dessas experiências musicais.

\section{Referências}

Agripino-Ramos, C. S., Lemos, E. L. D. M. D., \& Salomão, N. M. R. (2019). School Experiences and Autism Spectrum Disorder: What Do Children Say?. Revista Brasileira de Educação Especial, 25(3), 453-468.

Bieleninik, Ł., Geretsegger, M., Mössler, K., Assmus, J., Thompson, G., Gattino, G., \& Gold, C. (2017). Effects of improvisational music therapy vs enhanced standard care on symptom severity among children with autism spectrum disorder: the TIME-A randomized clinical trial. Jama, 318(6), 525-535.

Campos, L. K., \& Fernandes, F. D. M. (2016). School profile and language and cognitive abilities of children and adolescents with autism spectrum disorders. CoDAS, 28(3):234-43.

Chungpaibulpatana, J., Sumpatanarax, T., Thadakul, N., Chantharatreerat, C., Konkaew, M., \& Aroonlimsawas, M. (2008). Hyperbaric oxygen therapy in Thai autistic children. Medical journal of the Medical Association of Thailand, 91(8), 1232-8.

Crawford, M. J., Gold, C., Odell-Miller, H., Thana, L., Faber, S., Assmus, J., \& Hassiotis, A. (2017). International multicentre randomised controlled trial of improvisational music therapy for children with autism spectrum disorder: TIME-A study. Health Technology Assessment, 21(59), 1-40.

Ferreira, M. M. M., \& de França, A. P. (2017). O Autismo e as Dificuldades no Processo de Aprendizagem Escolar. Rev. Psicol, 11(38), 507-519.

Gattino, G.S. (2015). Musicoterapia e Autismo: teoria e prática. Memnon.

Le Menn-Tripi, C., Vachaud, A., Defas, N., Malvy, J., Roux, S., \& Bonnet-Brilhault, F. (2019). Sensory-Psychomotor evaluation in Autism: A new tool for functional diagnosis. L'encephale, 45(4), 312-319.

Lense, M. D., \& Camarata, S. (2020). PRESS-play: musical engagement as a motivating platform for social interaction and social play in young children with ASD. Music \& science, 3, 2059204320933080.

Lima, A. F. C., de Faria Gehres, A., Lorenzini, A. R., \& Brasileiro, L. T. (2017). A Influência de práticas pedagógicas e terapêuticas não verbais no transtorno do espectro autista: as possibilidades para o profissional de educação física. Motricidade, 13, 87-96.

Mapelli, L. D., Barbieri, M. C., Castro, G. V. D. Z. B., Bonelli, M. A., Wernet, M., \& Dupas, G. (2018). Child with autistic spectrum disorder: care from the family. Escola Anna Nery, 22(4):e20180116.

Mössler, K., Gold, C., Aßmus, J., Schumacher, K., Calvet, C., Reimer, S., \& Schmid, W. (2019). The therapeutic relationship as predictor of change in music therapy with young children with autism spectrum disorder. Journal of autism and developmental disorders, 49(7), 2795-2809.

Panerai, S., Ferri, R., Catania, V., Zingale, M., Ruccella, D., Gelardi, D., \& Elia, M. (2020). Sensory profiles of children with autism spectrum disorder with and without feeding problems: A comparative study in sicilian subjects. Brain Sciences, 10(6), 336.

Rabeyron, T., Del Canto, J. P. R., Carasco, E., Bisson, V., Bodeau, N., Vrait, F. X., \& Bonnot, O. (2020). A randomized controlled trial of 25 sessions comparing music therapy and music listening for children with autism spectrum disorder. Psychiatry Research, $293,113377$.

Lima Reis, D. D., Neder, P. R. B., da Conceição Moraes, M., \& Oliveira, N. M. (2019). Perfil epidemiológico dos pacientes com Transtorno do Espectro Autista do Centro Especializado em Reabilitação. Pará Research Medical Journal, 3(1), 1-8.

Rocha, C. C., Souza, S., de, M. V., Costa, A. F., \& Portes, J. R. (2019). O perfil da população infantil com suspeita de diagnóstico de transtorno do espectro autista atendida por um Centro Especializado em Reabilitação de uma cidade do Sul do Brasil. Physis: Revista de Saúde Coletiva, 29 , e290412.

Rossignol, D. A., \& Frye, R. E. (2012). A review of research trends in physiological abnormalities in autism spectrum disorders: immune dysregulation, inflammation, oxidative stress, mitochondrial dysfunction and environmental toxicant exposures. Molecular psychiatry, 17(4), 389-401.

Rossignol, D. A., \& Rossignol, L. W. (2006). Hyperbaric oxygen therapy may improve symptoms in autistic children. Medical hypotheses, 67(2), 216-228.

Rossignol, D. A., Rossignol, L. W., Smith, S., Schneider, C., Logerquist, S., Usman, A., \& Mumper, E. A. (2009). Hyperbaric treatment for children with autism: a multicenter, randomized, double-blind, controlled trial. BMC pediatrics, 9(1), 1-15.

Sakulchit, T., Ladish, C., \& Goldman, R. D. (2017). Hyperbaric oxygen therapy for children with autism spectrum disorder. Canadian Family Physician, 63(6), 446-448 
Research, Society and Development, v. 10, n. 4, e2010413868, 2021

(CC BY 4.0) | ISSN 2525-3409 | DOI: http://dx.doi.org/10.33448/rsd-v10i4.13868

Schmid, L., DeMoss, L., Scarbrough, P., Ripple, C., White, Y., \& Dawson, G. (2020). An Investigation of a Classroom-Based Specialized Music Therapy Model for Children With Autism Spectrum Disorder: Voices Together Using the VOICSS ${ }^{\mathrm{TM}}$ Method. Focus on Autism and Other Developmental Disabilities, 35(3), 176-185.

Sharda, M., Silani, G., Specht, K., Tillmann, J., Nater, U., \& Gold, C. (2019). Music therapy for children with autism: investigating social behaviour through music. The Lancet Child \& Adolescent Health, 3(11), 759-761.

Sharda, M., Tuerk, C., Chowdhury, R., Jamey, K., Foster, N., Custo-Blanch, M., \& Hyde, K. (2018). Music improves social communication and auditorymotor connectivity in children with autism. Translational psychiatry, 8(1), 1-13.

Tyler, K., MacDonald, M., \& Menear, K. (2014). Physical activity and physical fitness of school-aged children and youth with autism spectrum disorders. Autism research and treatment, 2014:e312163.

Volokitina, T. V., \& Ivanitskaya, E. Y. (2005). A Study of Spectral Characteristics of Cardiac Rhythm in Children of Primary School Age during Mental Work. Biomedical Engineering, 39(2), 75-76. 